\title{
Inflation for Bianchi IX models
}

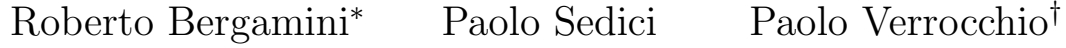

Istituto di Radioastronomia del C.N.R.,via Gobetti 101, 40100, Bologna, Italy

\begin{abstract}
The influence of Inflation on initial (i.e. at Planck's epoch) large anisotropy of the Universe is studied. To this end we consider a more general metric than the isotropic one: the locally rotationally symmetric (L.R.S.) Bianchi IX metric. We find, then, a large set of initial conditions of intrinsic curvature and shear allowing an inflationary epoch that make the anisotropy negligible. These are not trivial because of the non-linearity of the Einstein's equations.
\end{abstract}

\footnotetext{
*bergamini@astbo1.bo.cnr.it

†verrocchio@astbo1.bo.cnr.it
} 


\section{Introduction}

The observations of Cosmic Microwave Background Radiation can be utilized to give some constraints on the anisotropy of the Universe.

These limits have been derived analytically by Hawkings and Collins [1], considering little deviations from isotropy; whilst Barrow, Juszkiewicz and Sonoda [2] have computed the temperature pattern and the angular correlation function for the temperature perturbations expected in anisotropic models. Bunn, Ferreira and Silk have used the theoretical temperature pattern of the Bianchi model of type $V I I_{h}[3]$ to determinate the values of shear and vorticity making a best-fit with C.M.B.R. experimental data. A model independent approach at the problem has been introduced by Maartens, Ellis and Stoeger 四.

All this results are summarized in table (11) and they show that, actually, Universe is isotropic with a good approximation.

The set of initial conditions allowed from General Relativity is very larger than isotropy; so we must look for a physical process making Universe isotropic if, at Planck's epoch, it was highly anisotropic.

The theory of Inflation, as it leads to 'natural' prediction about the value of the curvature of the Universe, about the spectrum of scalar and tensor perturbation [12, 13, and it solves the topological defects, flatness and horizon problems [14, could be this physical process; even if it is not the only candidate $5,6,6,8,9$, 10, 11.

To verify this hypothesis we assume an anisotropic metric, the Bianchi metric [15. 16], and we introduce the stress-energy tensor of a scalar field minimally coupled to gravity that can give an Inflationary epoch 17, 18].

In this way, we are looking for the initial conditions allowing Inflation and study the evolution of anisotropy, comparing final values with the observed one.

Between the different Bianchi types, we studied Bianchi IX model, because it is the only one allowing positive intrinsic curvature $\stackrel{3}{R}[19$.

Using the Raychaudhuri relation 1

$$
\frac{2}{3} \theta^{2}=2 \frac{V(\phi)}{M_{P L}^{2}}+2 \sigma^{2}-\stackrel{3}{R}
$$

(where $V(\phi)$ is the potential energy of scalar field, $\theta$ measures the rate of expansion of the Universe and $\sigma$ is the shear of the homogeneous hypersurfaces [20)

it is clear that, in Bianchi IX model, the positive value of $\stackrel{3}{R}$, describing a closed Universe, could cancel the expansion of the volume of the Universe, even with a scalar field acting as cosmological constant [21, 22]; whereas in the other Bianchi types, open or flat, shear and intrinsic curvature 'help' the expansion.

The study of evolution of shear in Bianchi V model [23, 24] shows that Inflation leads this model to be completely isotropic.

\footnotetext{
${ }^{1}$ We will use such units that $c=h=k=1, \frac{8 \pi G}{3 c^{2}}=M_{P L}^{-2}$.
} 
An attempt to study the dynamics of Bianchi models with a scalar field, using the Nöther symmetries in minisuperspace, has been recently performed 25]; but there is not yet a complete analysis.

\section{Inflation with Bianchi IX metric}

Let us consider the metric of locally rotationally symmetric (L.R.S.) Bianchi IX Universe f: [26, 27, 28]:

$$
d s^{2}=-M_{P L}^{-2} N^{2}(\lambda) d \lambda^{2}+e^{-2 \alpha}\left[e^{-2 \beta}\left(\omega^{1}\right)^{2}+e^{\beta}\left(\left(\omega^{2}\right)^{2}+\left(\omega^{3}\right)^{2}\right)\right]
$$

where $N$ is the lapse function and the one-forms $\omega$ are defined by:

$$
d \omega^{i}=\frac{1}{2} \epsilon_{j k}^{i} \omega^{j} \wedge \omega^{k}
$$

The evolution's rate of $\beta$ is related to shear:

$$
\sigma^{2}=3\left(\frac{M_{P L}}{N} \beta^{\prime}\right)^{2}
$$

The general Bianchi IX model is more complicated with respect to L.R.S. Bianchi IX model; in fact in that case the Einstein's equations have not been yet solved. Our simplification does not affect the fundamental feature of the model that we want to study: the effect of positive curvature, and it allows us to compute the anisotropic initial conditions compatible with Inflation.

The Lagrangian for gravitational and scalar field is 29, 30, 31]:

$$
\begin{gathered}
L=M_{P L}^{2}\left(\frac{1}{2} w\left(-\alpha^{\prime 2}+{\beta^{\prime}}^{2}+\frac{1}{M_{P L}^{2}}{\phi^{\prime}}^{2}\right)-\frac{12}{w} e^{-4 \alpha} U(\beta)-\frac{24}{w} e^{-6 \alpha} \frac{V(\phi)}{M_{P L}^{4}}\right) \\
U(\beta)=-2 e^{-2 \beta}+\frac{1}{2} e^{-8 \beta}
\end{gathered}
$$

where the gauge freedom is hidden in the function $w \equiv \frac{12 e^{-3 \alpha}}{N(\lambda)}$.

The common feature to different theories of Inflation is the so-called 'slow-roll' approximation 32] in the description of motion of the field. This implies that Klein-Gordon equation reduces to:

$$
3 \frac{M_{P L}^{2}}{N^{2}} \alpha^{\prime} \phi^{\prime}=\frac{\partial V}{\partial \phi}
$$

With this approximation, models of Inflation satisfy two other conditions on potential $V(\phi)$ :

$$
\epsilon \equiv \frac{M_{P L}^{2}}{2}\left(\frac{1}{V} \frac{\partial V}{\partial \phi}\right)^{2} \ll 1
$$

\footnotetext{
2 The latin index $\mathrm{i}, \mathrm{j}$, etc $=1,2,3$.
} 


$$
\eta \equiv M_{P L}^{2} \frac{1}{V} \frac{\partial^{2} V}{\partial \phi^{2}} \ll 1
$$

As we are not interested in the length of Inflation but to initial conditions causing Inflation, it is enough to verify that the characteristic time in the evolution of scalar field $T_{\phi}$ is much greater than gravitational characteristic time $T_{G}$.

In our model $T_{G} \propto M_{P L}^{-1}$, whereas from assumptions (7) and (8) we obtain $T_{\phi} \propto\left(\frac{M_{P L}^{3}}{\epsilon V(\phi)}\right)$

We shall assume a value of about $M_{P L}^{4}$ for energy potential density at Planck's epoch in 'chaotic' Inflation theory. This assumption is justified by quantum effects giving radiative corrections to effective potential [33] of this typical order of magnitude [34], then:

$$
T_{\phi}=\frac{1}{\epsilon} T_{G}
$$

We can neglect, then, the 'kinetic' contribution of scalar field and assume that the potential term acts as a cosmological constant.

Hence, the contribution to lagrangian due to scalar field becomes:

$$
L_{\phi} \approx M_{P L}^{2} \frac{24}{w} e^{-6 \alpha}
$$

The solutions of the Einstein's equations in this case have been found by Cahen and Defrise [35]; following the work of Uggla,Jantzen and Rosquist we made a different choice of slicing gauge and we have found the solutions of Einstein's equations for the new functions:

$$
W=e^{-3 \alpha-3 \beta}, Z=e^{2 \alpha-2 \beta}
$$

They can be written in the following way:

$$
\begin{gathered}
Z(\tau)=\Gamma\left(1-\tau^{2}\right) \\
W(\tau)=\Gamma^{-\frac{5}{2}}\left\{\left(\Gamma-\frac{4}{3}\right)\left[8\left(1-\tau^{2}\right)^{\frac{1}{2}}-4\left(1-\tau^{2}\right)^{-\frac{1}{2}}\right]+\frac{4}{3}\left(1-\tau^{2}\right)^{-\frac{3}{2}}+\Sigma \tau\right\}+\Delta
\end{gathered}
$$

where $\Gamma, \Sigma$ and $\Delta$ are constants of integration.

Inserting the solutions (13)(14) in (11), we obtain the constraint $\Delta=0$.

The new coordinate time $\tau$ is related to comoving time $t$ by:

$$
d t=-2 M_{P L}^{-1} \Gamma^{\frac{1}{2}} W^{-\frac{1}{2}} Z^{-\frac{7}{4}} d \tau
$$

We see that when $\tau \rightarrow 1$ then $t \rightarrow-\infty$, and when $\tau \rightarrow-1$ then $t \rightarrow \infty$. 
We choose as initial comoving time $t_{P L}=10^{-43}$ sec.; the correspondence with initial coordinate time $\tau_{0}$, for a given solution of Einstein equations, depends on the choice of integration constant in (15).

The physically important part of the solutions is limited to interval $\left[\tau_{0},-1[\right.$, i.e. $\left[t_{P L},+\infty[\right.$.

The physical quantities $\theta$ and $\sigma$ can be expressed as functions of $W$ and $Z$ :

$$
\begin{gathered}
\theta=M_{P L} \Gamma^{-\frac{1}{2}}\left(\frac{3}{8} W^{\frac{1}{2}} Z^{\frac{3}{4}} \frac{d Z}{d \tau}-\frac{1}{4} W^{-\frac{1}{2}} Z^{\frac{7}{4}} \frac{d W}{d \tau}\right) \\
\sigma=M_{P L} \Gamma^{-\frac{1}{2}}\left|\frac{\sqrt{3}}{8} W^{\frac{1}{2}} Z^{\frac{3}{4}} \frac{d Z}{d \tau}+\frac{\sqrt{3}}{12} W^{-\frac{1}{2}} Z^{\frac{7}{4}} \frac{d W}{d \tau}\right|
\end{gathered}
$$

\section{Initial conditions of the Universe}

The initial coordinate time chosen is $\tau_{0}=0$; in this way by (12) and (16) we can write explicitly the relation between integration constants and the quantities $\beta_{0}$ and $\theta_{0}$ :

$$
\begin{gathered}
\Gamma=\frac{4}{4-e^{-6 \beta_{0}}} \\
\Sigma=-\frac{8 \theta_{0}}{M_{P L}} \sqrt{\Gamma-1}
\end{gathered}
$$

Because of (13) and (12), $\Gamma$ must be positive.

Equation (18) implies:

$$
\beta_{0}>-\frac{1}{6} \ln (4) \equiv \beta_{L I M}
$$

that, for the equality

$$
\stackrel{3}{R}=-M_{P L}^{2} e^{2 \alpha} U(\beta)
$$

is equivalent to:

$$
\stackrel{3}{R_{0}}>0
$$

The volume of the Universe is given by:

$$
\begin{gathered}
\text { Vol }=e^{-3 \alpha}=\frac{W^{\frac{1}{2}}}{Z^{\frac{3}{4}}} \\
=\frac{\sqrt{\left(\Gamma-\frac{4}{3}\right)\left[8\left(1-\tau^{2}\right)^{\frac{1}{2}}-4\left(1-\tau^{2}\right)^{-\frac{1}{2}}\right]+\frac{4}{3}\left(1-\tau^{2}\right)^{-\frac{3}{2}}+\Sigma \tau}}{\Gamma^{2}\left(1-\tau^{2}\right)^{\frac{3}{4}}}
\end{gathered}
$$


The behaviour of this function depends on the two independent initial conditions $\beta_{0}$ and $\theta_{0}$. The other two initial conditions $\alpha_{0}$ and $\sigma_{0}$ are related to $\beta_{0}$ and $\theta_{0}$ by:

$$
\begin{gathered}
-\alpha_{0}=-\beta_{0}+\frac{1}{2} \ln \Gamma \\
\sigma_{0}=\frac{\sqrt{3}}{3} \theta_{0}
\end{gathered}
$$

The former is not physically relevant because simply fixes the length scales at $\tau=0$. The latter, instead, is the consequence of Hamiltonian constraint (11), and it means that (with the choice $\tau_{0}=0$ ) our model describes an initial 'equipartition' of energy among the different gravitational degrees of freedom $\alpha$ e $\beta$. This is a more general and 'natural' initial condition than isotropy, and it has been recently analyzed by Barrow [36.

The function (23), for different choices of free parameters $\Gamma$ e $\Sigma$, gives two possible evolution of the Universe's volume. In the first case, the anisotropy cannot stop expansion, and we have;

$$
\mathrm{Vol}=e^{-3 \alpha} \rightarrow+\infty \text { when } \tau \rightarrow-1
$$

In the second case, the volume reaches a maximum and then it shrinks again, while shear and intrinsic curvature diverge; this happens at a coordinate time $\tau_{C} \in\left[0,-1\left[\right.\right.$, corresponding always to a finite comoving time $t_{C}$. Integrating numerically the equation $(15)$ we can show that $t_{C} \approx$ a few $t_{P L}$, and the exact value depends on initial condition $\Gamma$ and $\Sigma$.

The former possibility occurs if $\theta_{0}>\theta_{\star}$, where:

$$
\begin{gathered}
\left.\left.\theta_{\star} \approx 54.60 M_{P L} \quad \beta_{0} \in\right] \beta_{L I M}, .996 \beta_{L I M}\right] \\
\theta_{\star} \approx 0.05 M_{P L}\left(\ln \frac{\beta_{L I M}}{\beta_{L I M}-\beta_{0}}\right)^{3.80} \beta_{0} \in\left[.996 \beta_{L I M}, .792 \beta_{L I M}\right] \\
\theta_{\star} \approx 0 \quad \beta_{0} \in\left[.792 \beta_{L I M},+\infty[\right.
\end{gathered}
$$

otherwise Universe does not inflate.

Hence, it exits an initial value of $\beta_{0}=0.792 \beta_{L I M} \equiv \beta_{\star}$ distinguishing two different behaviours:

a) $\beta_{0}>\beta_{\star}$ There is Inflation with any initial value of expansion $\theta_{0}$ and shear $\sigma_{0}$, even if their values are much greater than $M_{P L}$.

To explain this unexpected result, let us come back to Lagrangian (5), obtaining the differential equation for the evolution of $\beta$ with respect to comoving time $t$ : 


$$
\frac{d^{2} \beta}{d t^{2}}-3 \frac{d \alpha}{d t} \frac{d \beta}{d t}+\frac{1}{12} M_{P L}^{2} e^{2 \alpha} \frac{\partial}{\partial \beta} U(\beta)=0
$$

then, we can show that, with high values of $\theta_{0}$ and $\sigma_{0}$, the 'friction' term:

$$
-3 \frac{d \alpha}{d t} \frac{d \beta}{d t}
$$

due to combined action of expansion and shear is more relevant than 'forcing' term:

$$
+\frac{1}{12} M_{P L}^{2} e^{2 \alpha} \frac{\partial}{\partial \beta} U(\beta)
$$

because the potential $U(\beta)$ is not enough steep, i.e. the value of derivative of potential with respect to $\beta$ is not enough large; moreover the large value of $\theta$ implies that $e^{2 \alpha}$ becomes 'small' very quickly.

After a few Planck's times the intrinsic curvature and the shear become dynamically negligible and they decay:

$$
\begin{gathered}
\sigma^{2}=\mathrm{Vol}^{-2} \\
\stackrel{3}{R}=\mathrm{Vol}^{-\frac{2}{3}}
\end{gathered}
$$

whereas expansion $\theta$ reaches his isotropic value $\sqrt{3} M_{P L}$ (equation(16)).

b) $\beta_{0}<\beta_{\star}$

This case is more complicated; in fact $\theta_{\star} \neq 0$, and it reaches the value of a few ten of Planck's mass when $\beta_{0} \rightarrow \beta_{L I M}$.

Universe inflate if $\theta_{0}>\theta_{\star}$, or, because of (25), if $\sigma_{0}>\frac{\sqrt{3}}{3} \theta_{\star}$. Hence, with respect to previous case, Inflation can be avoided; but, surprisingly, only with the smallest value of shear.

If there is Inflation, equations (31) (32) remain valid; again Universe becomes isotropic. If, instead, there is not Inflation, we have that:

$$
\beta_{+} \rightarrow \infty
$$

The equations (6) (17) (21), then, show that:

$$
\begin{gathered}
\sigma^{2} \rightarrow \infty \\
\stackrel{3}{R} \rightarrow \infty
\end{gathered}
$$

This behaviour is due to initial large 'steepness' of potential $U(\beta)$ when $\beta_{0}<\beta_{\star}$. This gives to $\beta$ an initial 'acceleration' enough to win the 'friction' term (29) and reach the values $\beta \gg 1$ 
In our model, the dependence of evolution of the volume on the initial value of intrinsic curvature $\stackrel{3}{R}_{0}$ is non trivial. For example, the relation (21) shows that when $\beta_{0} \rightarrow \beta_{L I M}$ the curvature $\stackrel{3}{R} \rightarrow 0$, whereas if $\beta_{0} \rightarrow \infty$ then $\stackrel{3}{R}_{0} \rightarrow 2 M_{P L}^{2}$. In the first case, where Universe is nearly flat at the beginning, there is Inflation only if $\theta_{0} \gg M_{P L}$, that is, because of (25), if its shear is very large. In the second case, where the energy tied to curvature is comparable with scalar field potential energy, Universe inflate for any value of $\theta_{0}$ and $\sigma_{0}$.

We must note that $\theta_{\star}$ falls suddenly at $\beta_{0} \approx \beta_{\star}$. In fact, for $\beta_{0} \leq \beta_{\star}$, the value of $\theta_{\star}$ is small $\left(\approx 10^{-3}\right)$ but finite, whereas for $\beta_{0}>\beta_{\star} \theta_{\star}$ is null. This is a consequence of strong non-linearity of Einstein's equations. Slightly different initial conditions can evolve in completely different way.

\section{Conclusions}

A strongly anisotropic Universe $(\theta \sim \sigma)$ at early times, can go through an Inflationary period, because of a scalar field minimally coupled to gravity. In this case, equations(31)(32) show that shear and curvature became negligible with respect the expansion. In fact the Inflation causes the growth of $10^{40}$ times, at least, of linear dimension of the Universe; this assure that $\sigma^{2}$ and $\stackrel{3}{R}$ decrease, respectively, of $10^{240}$ and $10^{80}$ times. The expansion $\theta$, instead, remains nearly constant. In this case, at the end of Inflation, the Universe can be described with the flat F.R.W. metric.

For $\beta_{0} \geq \beta_{\star}$ this situation happens for any initial value of $\sigma, \theta$ and $\stackrel{3}{R}$; hence, even if the anisotropy and curvature 'energy' are very larger than scalar field 'energy'. For $\beta_{0} \leq \beta_{\star}$, instead, Universe does not inflate only if $\theta_{0} \leq \frac{\sqrt{3}}{3} \theta_{\star}$.

The fundamental feature to underline is that the set of initial condition that do not allow inflation is small but finite

In the spirit of chaotic Inflation, we could assume that any form of energy at Planck's epoch be of Planck's energy order, $\sim M_{P L}$ (i.e. the energy 'equipartition' that Barrow has proposed). This would imply that Inflation would be avoided for $\beta_{0} \leq .883 \beta_{L I M}$, because $\theta_{\star} \sim M_{P L}$ at $\beta_{0} \sim .883 \beta_{L I M}$ and, when $\beta_{0} \rightarrow \beta_{L I M}$, it reaches to $54.60 M_{P L}$.

In this simple model, then, neither the initial values of dynamical quantities $\theta, \sigma$ nor the geometrical quantity $\stackrel{3}{R}$, would determine the evolution; the only important quantity would be $\beta$.

The authors are very grateful to dott.Francesco Chierici and dott.Carlo Bertoni for useful comments and suggestions. 


\section{References}

[1] S.W.Hawking, C.B.Collins, M.N.R.A.S. 142,129 (1969)

[2] J.D.Barrow, R.Juszkiewicz, D.H.Sonoda, M.N.R.A.S. 213,917 (1985)

[3] E.F.Bunn, P.Ferreira, J.Silk, submitted to Phy.Rev.Lett., e-Print Archive: astro-ph/9605123 (1996)

[4] R.Maartens, G.F.R.Ellis, W.R.Stoeger, Phys.Rev.D 51,5942 (1995)

[5] J.D.Barrow Q.Jl.R.astr.Soc. 23,344 (1982)

[6] C.W.Misner, Astrophysical J. 151,431 (1968)

[7] C.W.Misner Phys.Rev.Lett. 22,1071 (1969)

[8] C.W.Misner Phys.Rev 186,1319 (1969)

[9] S.W.Hawkins, C.Luttrell, Phys.Lett. 143B,83 (1984)

[10] R.Bergamini, G.Giampieri, Phys.Rev.D 40, 3960 (1989)

[11] V.A.Belinskij, I.M.Khalatnikov, E.M.Lifshitz, Adv.Phys. 19,525 (1970)

[12] A.R.Liddle, D.H.Lyth, Phys.Lett.B 291,391 (1992)

[13] A.R.Liddle, Phys.Rev.D 49,3805 (1994)

[14] Y.Hu, M.S.Turner, E.J.Weinberg, Phys.Rew.D 49,3830 (1994)

[15] M.A.H.MacCallum, General Ralativity: An Einstein centenary survey (Cambridge university press, Cambridge) (1979)

[16] G.R.F.Ellis, M.A.H.MacCallum, Commun. math. phys. 12,108 (1969)

[17] S.K.Blau, A.H.Guth, 300 years of gravitation (Cambridge university press, Cambridge) (1987)

[18] A.Linde, 300 years of gravitation (Cambridge university press, Cambridge) (1987)

[19] H.Sthephani, General Relativity: an introduction to the theory of the gravitational field (Cambridge university press, Cambridge) (1982)

[20] G.F.R.Ellis Proceeding of the international school of physics 'Enrico Fermi', course 47 (1971)

[21] R.M.Wald, Phys.Rev.D 29,2783 (1983)

[22] T.Rothman, G.F.R.Ellis, Phys.Lett.B 180,19 (1986) 
[23] A.B.Henriques, L.E.Mendes, General Relativity and Gravitation 26,61 (1994)

[24] E.Martinez Gonzalez, B.J.T.Jones, Phys.Lett. 167B,37 (1986)

[25] S.Capozziello, G.Marmo, C.Rubano, P.Scudellaro, submitted to Int.Jou.Mod.Phys.D, e-Print Archive: gr-qc/9606050 (1996)

[26] C.W.Misner, Phys.Rev. 186,1319 (1969)

[27] A.H.Taub, Ann.Phys. 53,472 (1951)

[28] M.P.Ryan, L.C.Shepley, Homogeneous Relativistic Cosmologies (Princeton Univ.Press, Princeton, New York) (1975)

[29] R.Arnowitt, S.Deser, C.W.Misner, Gravitation: An introduction to current research (J.Wiley \& sons, Inc., New York) (1962)

[30] M.P.Ryan, Hamiltonian Cosmology (W.Beiglböck, Heidelberg) (1972)

[31] C.Huggla, R.T.Jantzen, K.Rosquist, Phys.Rev.D 51,5522 (1995)

[32] A.Linde Phys.Lett. 106B,389 (1982)

[33] J.V.Narlikar, T.Padmanabhan, Gravity, Gauge Theories and Quantum Cosmology (D.Reidel publishing company, Dordrecht) (1986)

[34] A.Linde, Lectures on Inflationary Cosmology Proceeding of the ninth Lake Louise Winter institute, particle physics and cosmology (World scientific, Singapore) (1995)

[35] M.Cahen, L.Defrise, Commun.math,phys 11,56 (1968)

[36] J.D.Barrow, Phy.Rev.D 51,3113 (1995) 


\begin{tabular}{|c|c|c|c|c|c|}
\hline Model & & H.C. & B.J.S. & B.F.S & M.E.S. \\
\hline Bianchi I & $\left(\frac{\sigma}{\theta}\right)_{0}$ & $6 \cdot 10^{-8}$ & & & \multirow{8}{*}{$10^{-5}$} \\
\hline \multirow{2}{*}{ Bianchi V } & $\left(\frac{\sigma}{\theta}\right)_{0}$ & $1.5 \cdot 10^{-4}$ & & & \\
\hline & $\left(\frac{\omega}{\theta}\right)_{0}$ & $4 \cdot 10^{-4}$ & $1.7 \cdot 10^{-7}$ & & \\
\hline \multirow{2}{*}{ Bianchi $V I I_{0}$} & $\left(\frac{\sigma}{\theta}\right)_{0}$ & $\frac{1}{x} 10^{-6}$ & \multirow[b]{2}{*}{$3.2 \cdot 10^{-9}$} & & \\
\hline & $\left(\frac{\omega}{\theta}\right)_{0}$ & $\frac{2}{x} 10^{-6}$ & & & \\
\hline \multirow{2}{*}{ Bianchi $V I I_{h}$} & $\left(\frac{\sigma}{\theta}\right)_{0}$ & $\frac{2}{x} 10^{-3}$ & & $0.5 \cdot 10^{-9}$ & \\
\hline & $\left(\frac{\omega}{\theta}\right)_{0}$ & $\frac{1}{x} 10^{-3}$ & $1.2 \cdot 10^{-7}$ & & \\
\hline Bianchi IX & $\begin{array}{l}\left(\frac{\sigma}{\theta}\right)_{0} \\
\left(\frac{\omega}{\theta}\right)_{0}\end{array}$ & $\begin{array}{c}10^{-3} \\
10^{-11}\end{array}$ & $3.9 \cdot 10^{-13}$ & & \\
\hline
\end{tabular}

Table 1: LIMITS ON SHEAR ( $\sigma)$ AND VORTICITY ( $\omega)$ OF THE UNIVERSE FROM C.M.B.R OBSERVATIONS. In Bianchi VII $I_{0}$ and Bianchi $V I I_{h}$ models there is an adjustable parameter $x$; the ratio of the comoving length scale over which the orientation of the principal axes of shear change, to the present Hubble radius. In Bianchi VII $I_{h}$ and Bianchi $V$ open models, we chose the limits for models with $\Omega_{0}=0.1$. Hakwing, Collins (H.C.) calculated the upper limits on anisotropy in 1973, when the upper limits on C.M.B.R. temperature anisotropy were approximately three order of magnitude greater than values observed by C.O.B.E.; their results, after correction, become comparable with the other ones. Barrow, Juszkiewicz,Sonoda (B.J.S) studied only the rotation, while Bunn,Ferreira,Silk (B.F.S) limited to Bianchi VII $I_{h}$. Both studies do not give analytic expression of the limits with respect to parameter $x$, for the comparison we chose $x=1$ (the limits are those from the quadrupole anisotropy). The Maartens,Ellis,Stoeger (M.E.S) analysis, instead, does not depend on the model, and they have the same order of magnitude for shear and vorticity. 